\title{
Quality of drug prescription in primary health care facilities in Mwanza, north-western Tanzania
}

\author{
GIVENESS MAMBILE ${ }^{1,2}$, EVELINE KONJE², BENSON R. KIDENYA², DEOGRATIAS KATABALO² and KAROL J. \\ MARWA $^{2^{*}}$ \\ 'Ilemela District Hospital, P.O. Box 735, Mwanza, Tanzania \\ ${ }^{2}$ Catholic University of Health and Allied Sciences, P.O. Box 1464, Mwanza, Tanzania
}

\begin{abstract}
Background: Drug therapy can improve a patient's quality of life and health outcomes if only used properly. However, data on prescription quality at primary health care facilities in Tanzania is scanty. The objective of this study was to assess the quality of drug prescriptions in selected health care facilities in two districts of Mwanza, Tanzania.

Methods: This descriptive cross-sectional study was carried out in Ilemela and Nyamagana districts in Mwanza, Tanzania. The study included 20 randomly selected public and private health care facilities. A random sample of the patient records in each facility was included. From each patient record, the latest prescription was assessed. Information assessed and recorded included prescribing indicators, frequency of prescription error, various formulations and groups of drugs prescribed. Prescribers were interviewed on factors affecting prescribing practices.

Results: A total of 570 prescriptions were studied. The average number of drugs prescribed per encounter was 2.8. The percentage of encounters with an antibacterial agent prescribed was $33.8 \%$ and injections constituted $7.9 \%$ of cases. Prescriptions by generic name were $85.8 \%$ while $90.6 \%$ of drugs prescribed were from the national essential drug list. Antibacterial agents, analgesics and antimalarial agents accounted for $75.3 \%$ of all drugs prescribed. Of all prescriptions, 485 (85.1\%) had at least an error. A total of 1,177 errors were made. Errors of omissions accounted for the majority (99.3\%) of all errors. The most commonly observed missing prescription particulars were the drug strength (23.2\%) and patient weight (22.2\%). The average number of drugs per encounter was higher in private facilities (3.2) than in public facilities (2.4) $(P<0.0001)$. The range of drugs per prescription was also higher in private (1-7) than in public facilities (15) $(P<0.0001)$. There were statistically significant differences in the distribution of various drug groups prescribed between public and private facilities and between health centres and dispensaries $(P<0.001$ and 0.027 , respectively).

Conclusion: Prescribing of higher number of drugs than the WHO recommendations and overuse of antibiotics is still a problem at health care facilities in Mwanza Tanzania. The frequency of occurrence of prescription errors found during the study was considerably high.
\end{abstract}

Keywords: drug prescription, primary health care facilities, Tanzania

\section{Introduction}

Prescription is a written communication mechanism between a prescriber and a dispenser and ultimately extends to the patient. A high quality of drug prescriptions is important to achieve the best treatment outcome with drugs and other related products. The most carefully made therapeutic decision may not be useful if the prescription does not clearly communicate the intent of the prescriber to the dispenser (Sketris et al. 2011). The principles of rational drug use can be applied when the correct diagnosis is established, the appropriate drug is chosen, and the drug is prescribed with a suitable dosage and duration. However, unnecessary and incorrect prescription of drugs is still a serious problem and affects treatment costs worldwide(Organization 2004). More than $50 \%$ of all medicines are prescribed, dispensed or sold inappropriately worldwide, and $50 \%$ of patients fail to take them correctly(Organization 2004).

The occurrence of suboptimal prescribing such as under-dose, overdose and inappropriate use of drugs exists worldwide and has been associated with the increase in Adverse Drug Reactions (ADRs) which are significant causes of drug related hospital admission (Sketris et al. 2011). ADRs

\footnotetext{
*Correspondence: E-mail:carol_maro@yahoo.com
} 
are associated with increased morbidity and mortality rates, increased risk of microbial resistance, prolonged hospital stay and consequently an increased cost of disease management in patients (Kayombo et al. 2012, Mehta et al. 2008). Although these ADRs are the most common cause of patient injury, they are often preventable (Mehta et al. 2008). This suggests the need to study prescribing practices in Tanzania. On the other hand, irrational prescribing is characterized by polypharmacy and excessive use of antibiotics even for non-bacterial infections (Tamuno 2011). It also includes inadequate dosages; the use of injections when oral medication is more appropriate and prescriptions that contravene clinical guidelines.

In Tanzania, primary health care facilities (PHCFs) are the cornerstone of the Tanzania national health policy because they are often the first contact point that people have with the health system. Prescribers in PHCFs are usually non-physician clinicians, such as clinical officers, clinical assistants and/or nurses who are trained to attend to simple cases and refer complicated cases to hospitals (Kahabuka et al. 2011).

The World Health Organization/ International Network of Rational Use of Drugs (WHO/INRUD) drug use indicators are intended to measure prescribing performance in primary health care facilities in a reliable way and provide information to health care managers concerning drug use, prescribing practices and important aspects of patient care to compare or monitor facilities over time. In many countries these indicators have been tested in the field extensively and have been found to be relevant, easy to generate, measurable, valid, consistent, reliable, representative, sensitive to change, understandable and action oriented (Holloway and Green 2004). However, these indicators have limitations because they do not describe why there is a problem. WHO/INRUD primary health facility prescribing indicators that monitor prescribing practices comprise the followings: average number of medicines per encounter, percentage of medicines prescribed by generic name, percentage of encounters with an antibiotic prescribed, percentage of encounters with an injection prescribed and percentage of medicines prescribed which are from the essential medicines list or formulary list. Measuring prescription quality is the first step to improve the use of medicines. If it is known why prescribers act as they do and which factors are influencing them, it becomes possible to design effective interventions, help change in behaviour and correct the problem.

Irrational use of drugs occurs in all countries and causes harm to people (El Mahalli 2012). It includes excessive use of antibiotics and injections, the use of drugs not listed in the National Essential Drug List (NEDL) and the use of drugs of uncertain origin. These practices compromise the drug supply systems and the quality of health care services (Tamuno and Fadare 2012). Rational drug prescribing is regarded as the use of the least number of drugs to obtain the best possible effect in the shortest period and at a reasonable cost. The WHO has recommended that the average number of drug per prescription should range between 1.6 and 1.8 (Isah et al. 2008). In Africa, the average number of drugs prescribed per patient encounter is comparatively high indicating polypharmacy (Tamuno 2011). Medical errors and adverse events are very common and among them, the most prevalent involve medication prescribing (Garrouste-Orgeas et al. 2012). Errors are possible at any step of the care process from medication selection to drug administration. Numerous studies have shown that patients admitted to hospitals are harmed as a result of medication errors, the majority of which are errors in prescribing (Garrouste-Orgeas et al. 2012). The identification of these errors requires efficient reporting systems usually based on a combination of methods. These efficient reporting systems have not yet been established in most developing countries like Tanzania.

Prescribing can be regarded as a function of the patient, the prescriber and the environment (Spinewine et al. 2007). The systems in which people work have also been shown to contribute to prescription errors (Dean et al. 2002). Although these errors are common, they can be avoided hence can be important targets for improvement (Bates et al. 1995). Sources of prescription errors include: illegibly written orders, dispensing errors, calculation errors, monitoring errors and administration errors. In most cases, physicians, pharmacists and nurses are 
the ones involved in the occurrence of these medication errors (Mayo and Duncan 2004). The reasons for prescription errors can be knowledge-based errors, rule-based errors or skill-based errors [23]. In addition, communication errors can occur as a result of prescriber's receipt of incorrect information. This cannot be considered as the failure of the prescriber but of other individual such as patients or other health care workers who are not writing out that particular prescription (Tully et al. 2009). Dean et al suggested most mistakes in prescribing are made because of slips in attention or lack of prescribers' application of the relevant rules [20]. Skill-based slips or lapses are most common. Other errors are influenced by a risky work environment, physical and heavy workload, mental well-being and lack of knowledge [20]. Organizational factors identified are inadequate training and low perceived importance of prescribing (Dean et al. 2002). Absence of self-awareness of errors is also a contributing factor (Dean et al. 2002). This includes initial difficulty in remembering prescribing errors suggesting prescribers are not worried about errors (Tully et al. 2009).

Drug therapy can improve a patient's quality of life and health outcomes if only used properly. The occurrence of suboptimal prescribing of drugs is the cause of an increase in adverse drug reactions. Data on prescription quality at primary health care facilities (PHCFs) in Tanzania is scanty. The objective of this study was therefore, to assess the quality of drug prescriptions in selected PHCFs in Mwanza, Tanzania.

\section{Materials and Methods}

\section{Study area and design}

A descriptive cross-sectional study was carried in llemela and Nyamagana districts, Mwanza region, Tanzania. The two districts have a population of about 706,453 inhabitants. Both public and private health care facilities were sampled and included in the study. A sampling frame was used to select the PHCFs and a multi-stage random sampling procedure was carried out. Prescriptions were sampled from the health care facility level (as the primary sampling unit) and at the patient records level. A representative sample of the patient records was secured. From those records, the prescriptions were audited.

A total of twenty facilities were sampled (Organization 1993). Two strata containing both public and private PHCFs were obtained, one from Ilemela (containing 31 facilities) and another from Nyamagana district (containing 31 facilities) making a total of 62 PHCFs. To ensure allocation of appropriate number of health facility of each type, the facilities were listed and numbered within each stratum. Public facilities were first selected followed by the private facilities. Again, within each category (public and private facilities), health centres were arranged first and dispensaries second. A systematic sampling technique was carried out by applying a sampling fraction on each stratum to obtain a total of 20 facilities to be included in the study.

\section{Data collection procedures}

In the record keeping rooms of these facilities, the patient records were organized alphabetically and each was assigned a specific registration number. A random sample of the patient records in each facility was obtained by using a table of random numbers. From each patient record, the latest prescription was assessed using a prepared data collection form. Thirty (30) prescribing encounters were obtained from each PHCF as per WHO guideline (Organization 1993). To minimize bias, only 15 prescriptions were picked from PHCFs that have one prescriber.

Medical records of patients who at least at one particular time were prescribed drugs from selected PHCFs at llemela and Nyamagana districts were assessed. The latest prescription from each patient's records was assessed by using a data extraction form adapted from WHO (Organization 1993). Medical records of admitted patients were excluded from the study. This is because the average length of stay in the facility may be higher so additional drugs may be needed (Organization 1993, Sapkota et al. 2011). 
A data collection form adapted from WHO (1993) was used for collecting information from prescriptions in the private and public PHCFs on demographic data as well as prescription data. Regarding prescriptions, information assessed and recorded from each facility include: WHO/INRUD prescribing indicators, frequency of prescription errors of omission and commission, percentages of various formulations prescribed and percentages of various groups of drugs prescribed. Information on factors affecting prescribing practices was obtained through face to face interviews with prescribers from the selected health facilities. In order to ensure data quality, quality assurance and control were done through using data collection form adapted from WHO [1993](Organization 1993). All data sheets were counterchecked for accuracy and data was double entered before data analysis.

\section{Data analysis}

Data coding was done followed by entry using Statistical Package for Social Sciences (SPSS. 17). Data was analysed using STATA 11 (Statistical Corporation, College Station, Texas, US). Descriptive statistics was summarized using frequencies and percentages for categorical data and using means and standard deviation for continuous data. Student's t-test was used to compare the average number of drugs per encounter. The difference in the distribution of drugs groups prescribed and prescribing errors between different districts, health facility ownership and levels were done using Pearson's chi-square test. Variables with a P-value less than 0.05 were considered statistically significant.

\section{Ethical consideration}

Ethical approval was sought from the joint approval of Catholic University of Health and Allied Sciences/Bugando Medical Centre Ethical Review Board. Permission to carry out a study in the selected facilities was sought from the two District Councils.

\section{Results}

\section{Prescriptions}

This study included a total of 20 public and private facilities from llemela and Nyamagana districts. Of these, $5(25 \%)$ were health centres and $15(75 \%)$ were dispensaries. Half of facilities were from each district. Ten (50\%) PHCFs were publically owned and ten (50\%) were from private sector. A total of 570 prescription records from outpatients were selected. Of all the prescriptions, 270 (47.4\%) were from Ilemela and 300 (52.6\%) were from Nyamagana. About a quarter (26.3\%) prescriptions were from health centres and $73.7 \%$ were from dispensaries. There were an equal number of prescriptions from public and private facilities. Of the total 570 prescriptions, the number of drugs per encounter ranged from 1-7 drugs. Only 198 (34.7\%) of prescriptions contained 2 drugs. More than half of the prescriptions had more than 2 drugs (Table 1 ).

Table 1: Distribution of prescriptions with different number of drugs in health facilities

\begin{tabular}{lll}
\hline Number of drugs & Number of prescriptions & Percent of prescription \\
\hline 1 & 74 & 12.98 \\
2 & 198 & 34.74 \\
3 & 159 & 27.89 \\
4 & 89 & 15.61 \\
5 & 33 & 5.79 \\
6 & 13 & 2.28 \\
7 & 4 & 0.7 \\
\hline
\end{tabular}

There were a total of 1,574 drugs prescribed with an overall average number of drugs per encounter of 2.8 $\pm 1.2(1574 / 570)$. Prescriptions from Ilemela and Nyamagana districts had similar average number of drugs per encounter of $2.7 \pm 1.2$ (733/270) and 2.8 \pm 1.2 (841/300), respectively. 
The same average number of drugs per encounter was also observed from prescriptions of dispensaries and health centres. The average number of drugs per encounter was statistically higher $(3.2)$ in private than public facilities $(2.4)(P<0.0001)$. The average number of drugs per encounter ranged from 1.9 to 5.7 .

\section{Use of WHO/INRUD prescribing indicators}

Of the total 570 prescriptions, the average number of drugs per prescription was $2.8 \pm 1.2$. The percentage of drugs encountered with antibiotics and injections were $33.7 \%$ and $7.9 \%$, respectively. The percentage of drugs prescribed by generic name was $85.8 \%$ while that of drugs prescribed from Tanzanian National Essential Drug List (NEDL) was 90.6\% (Table 2). Regarding the percentage of encounter with antibiotics prescribed, the values in Ilemela and Nyamagana districts were $34.5 \%$ and $33.1 \%$, respectively. At health centres and dispensaries, the values were $31.4 \%$ and $34.6 \%$, respectively. With regard to public and private ownership, the values were $33.2 \%$ and $34.3 \%$ respectively (Table 2 ).

Table 2: Drug prescribing indicators

\begin{tabular}{lll}
\hline Prescribing indicator & Average \pm SD/Percent & WHO Standard value (\%) \\
\hline Average number of drugs per encounter & $2.8 \pm 1.2$ & $1.6-1.8$ \\
Percentage encounter with antibiotics prescribed & 33.7 & $20.0-26.8$ \\
Percentage encounter with injections prescribed & 7.9 & $13.4-24.1$ \\
Percentage of drugs prescribed by generic name & 85.8 & 100 \\
Percentage of drugs prescribed from the NEDL & 90.6 & 100 \\
\hline
\end{tabular}

\section{Drug formulations}

Of the total 1,574 drugs prescribed, the most dominant formulations were tablets (56.7\%) and the least prevalent were other drugs formulations (0.3\%) such as suppositories, pessaries and soaps. However, the formulations of 80 drugs (5.0\%) were not indicated in prescriptions (Table 3 ).

Table 3: The percentage of prescribed drugs formulations

\begin{tabular}{lcc}
\hline Formulation & Frequency & Percentage \\
\hline Tablets & 892 & 56.7 \\
Syrups & 347 & 22.1 \\
Injections & 124 & 7.9 \\
Capsules & 95 & 6.0 \\
Creams and ointments & 32 & 2.0 \\
Others & 4 & 0.3 \\
Unknown & 80 & 5.0 \\
Total & $\mathbf{1 , 5 7 4}$ & $\mathbf{1 0 0}$ \\
\hline
\end{tabular}

\section{Drug groups}

Regarding drug groups of the 1574 drugs prescribed, the most prevalent were the antibacterial agents (33.8\%). The groups of $8(0.3 \%)$ drugs were not identified. There were statistically significant differences in the distribution of various drug groups prescribed between public and private facilities; and between health centres and dispensaries ( $P<0.001$ and 0.027 , respectively) (Table 4). Of the 570 prescriptions, $74.4 \%$ had at least one antibacterial drug prescribed. Also $99(86.1 \%)$ of 115 prescriptions with no diagnosis indicated had antibiotics prescribed. 
Table 4: The percentages of various drug groups by facility ownership and facility level

\begin{tabular}{|c|c|c|c|c|c|}
\hline \multirow[t]{2}{*}{ Drug group } & \multicolumn{2}{|c|}{ Facility ownership } & \multicolumn{2}{|l|}{ Type of facility } & \multirow[t]{2}{*}{ Total (\%) } \\
\hline & Public (\%) & Private $(\%)$ & Health Centre & Dispensary & \\
\hline Antibacterials & $223(33.1)$ & $307(34.1)$ & $131(31.3)$ & $399(34.5)$ & $530(33.8)$ \\
\hline Analgesics & $181(29.9)$ & $216(24.0)$ & $102(24.3)$ & $295(25.5)$ & $397(25.3)$ \\
\hline Antimalarials & $90(13.4)$ & $165(18.3)$ & $74(17.7)$ & $181(15.7)$ & $255(16.2)$ \\
\hline Liquid correcting electrolytes & $47(7.0)$ & $66(7.3)$ & $26(6.2)$ & $87(7.5)$ & $113(7.2)$ \\
\hline Antihypertensives & $22(3.3)$ & $32(3.6)$ & $20(4.8)$ & $34(3.0)$ & $54(3.4)$ \\
\hline Antispasmodics & $24(3.6)$ & $23(2.6)$ & $18(4 \cdot 3)$ & $29(2.5)$ & $47(3.0)$ \\
\hline Antiepileptic/Anticonvulsants & $21(3.1)$ & $22(2.5)$ & $8 \quad(1.9)$ & $35(3.0)$ & $43(2.7)$ \\
\hline Cough syrups & $14(2.1)$ & $30(3.3)$ & $9 \quad(2.2)$ & $35(3.0)$ & $44(2.8)$ \\
\hline Antihelminthes & $29(4.3)$ & $9(1.0)$ & $7 \quad(1.7)$ & $31(2.7)$ & $38(2.4)$ \\
\hline Vitamins/Minerals & $2(0.3)$ & $5(0.6)$ & $3(0.7)$ & $4 \quad(0.4)$ & $7(0.5)$ \\
\hline Antifungals & $0 \quad(0.0)$ & $9 \quad(1.0)$ & $4 \quad(1.0)$ & $5 \quad(0.4)$ & $9(0.6)$ \\
\hline Anti-inflammatories & $1 \quad(0.6)$ & $3(0.3)$ & $1 \quad(0.2)$ & $3(0.3)$ & $4(0.3)$ \\
\hline Antiacids & $1 \quad(0.2)$ & $3(0.3)$ & $3(0.7)$ & $1 \quad(0.1)$ & $4(0.3)$ \\
\hline Antidiabetics & $1 \quad(0.2)$ & $2(0.2)$ & $2(0.5)$ & $1 \quad(0.1)$ & $3(0.2)$ \\
\hline Others & $13(1.9)$ & $5 \quad(0.6)$ & $9 \quad(2.2)$ & $9(0.8)$ & $18(1.2)$ \\
\hline Unknown & $4 \quad(0.6)$ & $4 \quad(0.4)$ & $2 \quad(0.5)$ & $6 \quad(0.5)$ & $8(0.3)$ \\
\hline Total & 673 & 901 & 419 & 1,155 & 1574 \\
\hline P-value & $<0.001$ & & 0.027 & & \\
\hline
\end{tabular}

\section{Prescription errors}

Among all prescriptions observed, $485(85.1 \%)$ had at least one or more errors. A total of 1,177 errors were detected. Error of omission was the commonest, accounting for 1,169 (99.3\%) of all errors. There were only $8(0.7 \%)$ errors of commissions distributed in eight prescriptions. Of the eight prescriptions that had error of commission, $7(87.5 \%)$ of them had both errors of commission and omission. There were 485 (85.0\%) prescriptions with omissions and of these, 477 (98.6\%) had omissions alone. Of the 1,169 omissions made, the most common were the drug strength $(23.2 \%)$ and the patient weight (22.2\%). Name of patient was the least omitted particular and it accounted only $0.6 \%$ of all omissions (Table 5 ).

Table 5: Frequency of occurrence of errors of omission

\begin{tabular}{lll}
\hline Omission & Frequency & Percent \\
\hline Drug strength & 271 & 23.2 \\
Patient weight & 260 & 22.2 \\
Generic name of drug & 113 & 9.7 \\
Diagnosis & 112 & 9.6 \\
Address of patient & 103 & 8.8 \\
Age of patient & 89 & 7.6 \\
Signature of prescriber & 79 & 6.8 \\
Dosage form & 65 & 5.6 \\
Duration of treatment & 55 & 4.7 \\
Date & 14 & 1.2 \\
Name of patient & 8 & 0.6 \\
Total & $\mathbf{1 , 1 6 9}$ & $\mathbf{1 0 0 . 0}$ \\
\hline
\end{tabular}

Of the 8 errors of commission made, the prescribers committed the following errors: wrong strength 4 (50.0\%), ignoring drug-drug interaction, 3 (37.5\%) and wrong dosage 1 (12.5\%). The distribution of prescriptions with errors between public (84.9\%) and private $(85.3 \%)$ facilities was similar $(P=0.908)$. It was also similar between health $(87.3 \%)$ centres and dispensaries $(84.3 \%)(P=$ $0.368)$. 


\section{Factors affecting prescribing practices in the study area}

A total of 36 prescribers from 20 facilities were included in this study. Of these, $14(38.9 \%)$ prescribers were from health centres while 22 (61.1\%) were from dispensaries. Among the prescribers only $13(28.3 \%)$ reported to have working experience above 10 years. The professional level of prescribers was medical officers 2 (5.6\%), assistant medical officers $2(5.6 \%)$, clinical officers $26(72.2 \%)$ and assistant clinical officers $6(16.6 \%)$. Among the prescribers, only $27.8 \%$ knew about the existence of WHO standards on prescribing practice. The most common factors affecting prescribing practices was high patient work load 24 (25.8\%), lack of knowledge $13(14 \%)$ and influence from patients $19(20.4 \%$ ) (Table 6$)$.

Table 6: Factors affecting prescribing practices

\begin{tabular}{lcc}
\hline Factor & Frequency & Percentage \\
\hline High patient work load & 24 & 25.8 \\
Influence from patients & 19 & 20.4 \\
Lack of knowledge & 13 & 14.0 \\
Negligence & 10 & 10.8 \\
Availability of drugs & 9 & 9.7 \\
Business related factors & 8 & 8.6 \\
Lack of diagnostic and prescribing tools & 6 & 6.5 \\
Resistance and side effects of drugs & 4 & 4.3 \\
\hline
\end{tabular}

Only few prescribers (10.5\%) discuss and reach consensus with the dispenser when the dispenser report to errors of omission or commission. About two thirds (65.8\%) of the prescribers make corrections while about a quarter (23.7\%) do nothing.

\section{Discussion}

The prescribing indicators were developed by WHO with the intention to measure the degree of polypharmacy, the overall level of use of antibiotics and injections, the tendency to prescribe drugs by generic name and the prescribing practice based on the national essential drug list or standard treatment guideline (Angamo et al. 2011). The indicators are measured based on the prescribed drugs regardless of whether a patient received the drugs or not. These indicators are ultimately useful in promoting rational drug use. The average number of drugs per encounter from this study was slightly higher than the reported in Saudi Arabia, Malawi and Kenya (Charles 2014, El Mahalli 2012, Sosola 2007) and lower than reported in Nigeria and Ghana (Afriyie and Tetteh 2014, Chima et al. 2012). The differences in the average numbers of drugs per encounter may be caused by the differences in disease patterns in those countries. In general, the average number of drugs in the above African countries is higher than the WHO standard value (1.6-1.8) (Isah et al. 2008) indicating polypharmacy is common in this region. Lack of diagnostic tools may contribute to this practice.

Private facilities had a higher average number of drugs per encounter than the public ones which is similar to observations in Pakistan (Siddiqi et al. 2002). This shows that the level of polypharmacy is higher in private facilities than in public ones. This finding might be caused by the commercial orientation of health care services provided by the private facilities, whereby some of the patients were prescribed drugs for the indication shown plus other drugs whose indications were not provided. The higher average number of drugs per encounter in private facilities may also be due to the availability of more drugs in those facilities compared to the public ones. Although the results show that the private facilities practice polypharmacy to a larger extent than the public ones, it was noted that at least two of the facilities with the lowest average number of drugs per encounter belong to the former group.

Percentage encounter with antibiotics prescribed of $33.7 \%$ is higher than the standard established by WHO/INRUD (20.0-26.8\%) (Isah et al. 2008). Findings of this study are comparable to those reported from studies in Saudi Arabia and Nigeria (El Mahalli 2012, Tamuno and Fadare 
2012) but lower than in Ethiopia, Malawi, Niger and Zambia (Desalegn 2013, Hangoma 2015, Mallet et al. 2000, Sosola 2007). There was a large number of prescriptions with no diagnosis indicated, however the majority had antibacterial agents prescribed. This highlights that, there is an overuse of antibacterial agents whereby patients are given antibacterial drugs even for non-bacterial infections or for a prescription without the diagnosis indicated.

Surprisingly, Injections were prescribed in a lower percentage than WHO/INRUD standards which is uncommon in developing countries (Ismaeilzadeh et al. 2006). This finding is beneficial since overuse of injections is not encouraged because it carries risks such as sepsis at administration, tissue toxicity from local irritation, higher costs, difficulties in correcting the prescribing error and patient's infection by virus like Hepatitis C and HIV (Angamo et al. 2011). However the rate was relatively higher than reported in Nigeria (Tamuno and Fadare 2012) and much lower than in Ethiopia, Niger and Malawi (Desalegn 2013, Mallet et al. 2000, Sosola 2007). These findings support facts that, there is injection over use in developing countries owing to the belief by health professionals and lay people that injections are very powerful method for restoring or maintaining health (Ismaeilzadeh et al. 2006). Another study in Tanzania had reported a higher figure of $19 \%$ (Massele et al. 2001) than this study. This might be due to the differences in disease patterns between the two study areas and time between the current study and the other done 13 years ago assuming the prescribing practice has improved in Tanzania over time.

The proportion of drug prescribed by generic name in this study was lower than the WHO standard value (100\%) and reports from Malawi and Niger (Mallet et al. 2000, Sosola 2007). However it was higher than the findings in Ghana, Nigeria, Kenya and Ethiopia (Adebayo and Hussain 2010, Afriyie and Tetteh 2014, Charles 2014, Desalegn 2013, Massele et al. 2001, Tamuno and Fadare 2012). Prescribing by using non-generic names increases unnecessary costs to the patient by buying a brand drug while an equally effective cheaper generic drug can be obtained. Sometimes a patient may miss a drug in the community or private pharmacies and delay the treatment simply because the prescribed brand or trade name may not be familiar to the dispenser. This may worsen the prognosis of a particular disease thus jeopardising the public health if that disease is communicable.

The percent of drugs prescribed from the national essential drug list (NEDL) was $90.6 \%$, which is slightly lower than the ideal value of $100 \%$ established by WHO. The percent was also lower than findings of studies in Ethiopia and Malawi (Desalegn 2013, Sosola 2007) but similar to that reported from elsewhere in Tanzania (Massele et al. 2001). The similarity of the findings in Tanzania is likely to be attributed to the fact that public health facilities use Medical Stores Department as their main source of pharmaceuticals. A similar situation has been reported from Kenya (Charles 2014).

The high use of antimalarial agents, observed in this study, may be attributed to the common practice of presumptive treatment of all fevers with antimalarial agents in the study area(Msaki et al. 2012). Moreover, a higher figure of analgesics compared to antimalarial agents is expected since analgesics might be prescribed for malaria fever and non-malaria fever, for pain relief and for other inflammatory conditions. Antibacterial agents prescribed were higher than WHO standards in both private and public facilities. Antibacterial and analgesics being among the most prescribed drugs matches with findings from Ethiopia(Desalegn 2013). Moreover the value of prescribed antihypertensives in this study is lower than in Nigeria (Fadare et al. 2013, Tamuno and Fadare 2012). This might be explained by the inclusion of elderly patients in the Nigerian studies, who in most cases suffer from hypertension more commonly compared to other age groups unlike in this study where prescriptions were picked randomly regardless of the age of the patient.

This study identified that the majority of the prescriptions had at least one or more errors. This finding is lower than in Nigeria (Tamuno and Fadare 2012). The strength of the drug and the weight of the patient were the most commonly missing particulars. These accounted for about a fifth of the omissions. The absence of strength of drug in prescription leaves a room for the 
prudence of a dispenser to act while it may happen that the dispensed strength may differ from the one intended by a prescriber. Omission of weight of patient can cause discrepancies in dosage calculations and administration and might cause ADRs to a patient. Surprisingly, only few prescribers do discuss and reach consensus with the dispenser(s) when the later report about prescription errors. The dispenser contacts the patient at exit point and provides the necessary information to the patients who are the end users of the drug(s). Lack of proper communication between the prescriber and dispenser has a negative impact as afar as compliance, rational drug use and patient safety are concerned. There is a need for intervention by policy makers to address this gap between prescribers and dispensers. The most common factors affecting prescribing practices was high patient work load and influence from patients. This is not surprising due to high patient load reported in Sub-Saharan countries (Fatti et al. 2011).

In conclusion, Polypharmacy is a problem at primary health care facilities and is more common in the private than in the public PHCFs. The average number of drugs per encounter is generally higher than the WHO recommended value and is much observed in private than in public health care facilities. The frequency of occurrence of prescription errors is also predominantly high. In general, there is poor quality of drug prescriptions in primary health care facilities in Mwanza. There is a need for the policy makers and other stakeholders to enforce and promote rational prescribing by health care providers through adherence to national and international standards and prescribing indicators.

\section{Competing interests}

The authors declare that they have no competing interests.

\section{Authors' contributions}

$\mathrm{KJ}$ participated in proposal development,data collection,data analysis, manuscript drafting and submitting. GM participated in proposal development, data collection, data analysis and manuscript drafting. EK, BRK and DK participated in proposal development, data analysis and manuscript revising and approval for publication.

\section{Acknowledgements}

We sincerely thank nurses at the PHCFs for their valuable support with retrival of records, the Catholic University of Health and Allied Sciences and Regional Medical Officer for granting us a permission to carry the study.

\section{References}

Adebayo, E. \& Hussain, N. (2010) Pattern of prescription drug use in Nigerian army hospitals. Annals of African medicine, 9(3).

Afriyie, D. \&Tetteh, R. (2014) A description of the pattern of rational drug use in Ghana Police Hospital. Int J Pharm Pharmacol, 3, 143-148.

Angamo, M. T., Wabe, N. T. \& Raju, N. (2011) Assessment of patterns of drug use by using World Health Organization's prescribing, patient care and health facility indicators in selected health facilities in Southwest Ethiopia.

Bates, D. W., Cullen, D. J., Laird, N., Petersen, L. A., Small, S. D., Servi, D., Laffel, G., Sweitzer, B. J., Shea, B. F. \& Hallisey, R. (1995) Incidence of adverse drug events and potential adverse drug events: implications for prevention. JAMA : the journal of the American Medical Association, 274(1), 29-34. 
Charles, N. M. (2014) Patterns of prescribing practices in Makueni County Referral Hospital, Kenya. Unpublished, University of Narobi.

Chima, I. E., Obidiya, O. S. \& Abraham, C. V. M. (2012) Evaluation of Drug Use and Patient Care Practices in a Referral Health Facility in Yenagoa, Bayelsa State, Nigeria. Continental Journal of Pharmaceutical Sciences, 6(1), 10-6.

Dean, B., Schachter, M., Vincent, C. \& Barber, N. (2002) Causes of prescribing errors in hospital inpatients: a prospective study. The Lancet, 359(9315), 1373-1378.

Desalegn, A. A. (2013) Assessment of drug use pattern using WHO prescribing indicators at Hawassa University teaching and referral hospital, south Ethiopia: a cross-sectional study. $B M C$ health services research, 13(1), 170.

El Mahalli, A. (2012) WHO/INRUD drug prescribing indicators at primary health care centres in Eastern province, Saudi Arabia. EMHJ, 18(11).

Fadare, J. O., Agboola, S. M., Opeke, O. A. \& Alabi, R. A. (2013) Prescription pattern and prevalence of potentially inappropriate medications among elderly patients in a Nigerian rural tertiary hospital. Therapeutics and clinical risk management, 6, 115.

Fatti, G., Grimwood, A., Mothibi, E. \& Shea, J. (2011) The effect of patient load on antiretroviral treatment programmatic outcomes at primary health care facilities in South Africa: a multicohort study. JAIDS Journal of Acquired Immune Deficiency Syndromes, 58(1), e17-e19.

Garrouste-Orgeas, M., Philippart, F., Bruel, C., Max, A., Lau, N. \& Misset, B. (2012) Overview of medical errors and adverse events. Ann Intensive Care, 2(1), 2.

Hangoma, J. M. (2015) Antibiotic prescribing patterns among physicians at the University Teaching Hospital in Lusaka, Zambia.

Holloway, K. and Green, T. (2004) Drug and therapeutics committees. A practical guide World Health Organization, Geneva: 2003: WHO/EDM/PAR.

Isah, A., Ross-Degnan, D., Quick, J., Laing, R. \& Mabadeje, A. (2008) The development of standard values for the WHO drug use prescribing indicators. Geneva: WHO.

Ismaeilzadeh, A., Nikfar, S. \& Rahimi, W. (2006) Physicians' attitude toward injectable medicines. Journal of Pharmacology and Toxicology, 1(1), 33-39.

Kahabuka, C., Kvåle, G., Moland, K. M. \& Hinderaker, S. G. (2011) Why caretakers bypass Primary Health Care facilities for child care-a case from rural Tanzania. BMC health services research, 11(1), 315.

Kayombo, E. J., Uiso, F. C. \& Mahunnah, R. L. (2012) Experience on healthcare utilization in seven administrative regions of Tanzania. Journal of ethnobiology and ethnomedicine, 8, 5 .

Mallet, H., Njikam, A. \& Scouflaire, S. (2000) Evaluation of prescription practices and of the rational use of medicines in Niger]. Sante (Montrouge, France), 11(3), 185-193.

Massele, A., Nsimbi, S. \& Rimoy, G. (2001) Prescribing habits in church-owned primary health care facilities in Dar-Es-Salaam and other Tanzanian coast Regions. East African medical journal, 78(10), 510-514.

Mayo, A. M. \& Duncan, D. (2004) Nurse perceptions of medication errors: what we need to know for patient safety. Journal of nursing care quality, 19(3), 209-217.

Mehta, U., Durrheim, D. N., Blockman, M., Kredo, T., Gounden, R. \& Barnes, K. I. (2008) Adverse drug reactions in adult medical inpatients in a South African hospital serving a community with a high HIV/AIDS prevalence: prospective observational study. British journal of clinical pharmacology, 65(3), 396-406.

Msaki, B. P., Mshana, S. E., Hokororo, A., Mazigo, H. D. \& Morona, D. (2012) Prevalence and predictors of urinary tract infection and severe malaria among febrile children attending Makongoro health centre in Mwanza city, North-Western Tanzania. Archives of Public Health, 70(1), 1.

Organization, W. H. (1993) How to investigate drug use in health facilities: selected drug use indicators. 
Organization, W. H. (2004) Rational use of medicines by prescribers and patients. Executive Board. 115th Session. Provisional Agenda item, 4, 16.

Sapkota, S., Pudasaini, N., Singh, C. \& GC, S. (2011) Drug prescribing pattern and prescription error in elderly: A retrospective study of inpatient record. Asian Journal of Pharmaceutical and Clinical Research, 4(3), 129-132.

Siddiqi, S., Hamid, S., Rafique, G., Chaudhry, S., Ali, N., Shahab, S. \& Sauerborn, R. (2002) Prescription practices of public and private health care providers in Attock District of Pakistan. The International journal of health planning and management, 17(1), 23-40.

Sketris, I., Fisher, J., Langille, I. E., Bergman, U., Andersen, M. \& Vlahović-Palčkevski, V. (2011) Prescribing indicators: what can Canada learn from European countries? Journal of population therapeutics and clinical pharmacology= Journal de la therapeutique des populations et de la pharamcologie clinique, 19(1), e78-98.

Sosola, A. G. (2007) An assessment of prescribing and dispensing practices in public health facilities of southern Malawi. Unpublished, UNIVERSITY OF MALAWI.

Spinewine, A., Schmader, K. E., Barber, N., Hughes, C., Lapane, K. L., Swine, C. \& Hanlon, J. T. (2007) Appropriate prescribing in elderly people: how well can it be measured and optimised? The Lancet, 370(9582), 173-184.

Tamuno, I. (2011) Prescription pattern of clinicians in private health facilities in Kano, Northwestern Nigeria. Asian Pacific Journal of Tropical Disease, 1(3), 235-238.

Tamuno, I. \& Fadare, J. O. (2012) Drug prescription pattern in a Nigerian Tertiary Hospital. Tropical Journal of Pharmaceutical Research, 11(1), 146-152.

Tully, M. P., Ashcroft, D. M., Dornan, T., Lewis, P. J., Taylor, D. \& Wass, V. (2009) The causes of and factors associated with prescribing errors in hospital inpatients. Drug safety, 32(10), 819836. 